\title{
Single amino acid based self-assemblies of Cysteine and Methionine
}

\author{
Nidhi Gour* ${ }^{a}$, Bharti Koshti ${ }^{a}$, Chandra Kanth P. ${ }^{b}$ Dhruvi Shah ${ }^{c}$, Vivek Shinh Kshatriya ${ }^{a}$, Reena Agrawal-Rajput ${ }^{c}$, Manoj Kumar \\ Pandey \\ ${ }^{a}$ Department of Chemistry, Centre of Engineering and Enterprise, Institute of Advanced Research, Gandhinagar, Gujarat, India \\ ${ }^{b}$ Department of Science, School of Technology, Pandit Deendayal Petroleum University, Gandhinagar, Gujarat, India \\ ${ }^{c}$ School of Biological Science and Bioengineering, Institute of Advanced Research, Gandhinagar, Gujarat, India
}

\begin{abstract}
We report for the very first time self assembly of Cysteine and Methionine to discrenible strucutres under neutral condition. The nature of interactions which lead to such selfassemblies was purported by coincubating assemblies in urea and mercaptoethanol. Further interaction of aggregates with short amyloidogenic dipeptide diphenylalanine (FF) was assessed. While cysteine aggregates completely disrupted FF fibres, methionine albeit triggered fibrillation. The results presented in the manuscript are striking, since to the best of our knowledge,this is the first report which demonstrates that even non-aromatic amino acids (cysteine and methionine) can undergo spontaneous self-assembly to form ordered aggregates.
\end{abstract}

\section{Introduction}

Peptides have been the most attractive template for designing novel nanostructures due to their varied chemical and functional diversity. There are numerous reports wherein peptides has been used for designing smart nanomaterials with diverse applications. ${ }^{1}$ Self-assembly of peptides to nanotubes and nanofibers is of peculiar interest, due to its potential nano(bio)technology applications ranging from designed synthetic ion channels and tissue engineering to bioelectronics, optical and spintronic materials. ${ }^{2}$ The pioneering work of Reches and Gazit demonstrated that aromatic dipeptide diphenylalanine (FF) self-assemble to rigid nanotubular structures and further elucidated its application as scaffolds for casting silver nanowire. ${ }^{3}$ This was a breakthrough research which led to significant interest/efforts for assessing selfassembling behaviour of short peptides. ${ }^{4}$ More importantly, it was elucidated that FF sequence is present at 19 and 20 position of $A \beta(1-42)$ sequence and plays a crucial role in aggregation of the amyloidogenic protein. ${ }^{5}$ In yet another very interesting study, Adler Abromovick and coworkers for the very first time reported self-assembling property of single amino acid phenylalanine by various biophysical and cell-based assays. ${ }^{6}$ They reported the assemblies of phenylalanine to be toxic in nature with amyloid characteristics, thus relating the etiology of phenylketonuria to amyloid associated disease. Henceforth, the study of self-assembling properties of individual aminoacid has become a topic of crucial interest for understanding the etiology of diseases caused by protein aggregation like Alzheimer's and Parkinson's. Perween and coworkers further studied self-assembled fibres formed by single amino acids phenylalanine and tyrosine by various techniques like circular dichroism, FTIR, Congo red and thioflavin $\mathrm{T}$ assays and elucidated that the structures were 1D assemblies lacking any secondary structure like $\alpha$-helix and $\beta$ sheet and even purported the nature of interactions to be simply non covalent pi-pi interactions. $^{7}$ A recent report also indicate that single amino acids tryptophan and tyrosine form fluorescent nanotubes as assessed by fluorescence microscopy studies. ${ }^{8}$ More significantly, single amino acid based self-assembled structures also provide a very simple and facile 
methodology for design of novel nanomaterials with diverse applications in materials and biology. ${ }^{9}$

As a consequence of these studies, and our previous research interest on bio-molecular selfassembly, ${ }^{10}$ we were motivated to examine the self-assembling propensity of individual amino acids in solution to get further insights into its structure formation. Herein, we present for the very first time a comprehensive study of self-assembling behaviour of 21 L-amino acids in aqueous solution. Of the $21 \mathrm{~L}$-amino acids studied, only five amino acids namely cysteine (Cys), methionine (Met), tyrosine (Tyr), phenylalanine (Phe), and tryptophan (Trp), showed spontaneous self-assemblies as visualised by optical microscopy in neutral aqueous solution. While it has been previously reported that tyrosine, tryptophan and phenylalanine do assemble to fibre/nanotube like structures, self-assembling propensity of Cys and Met is still unknown. Hence, the results presented in the manuscript are significant, since they demonstrate for the very first time, spontaneous self-assembly of non-aromatic amino acids like Cys and Met to discernible structures in solution. The self-assembling behaviour of Cys was particularly peculiar since it revealed a neuronal fibril like morphology with fibres appearing from single focus (Figure 1, Fig. S2, ESI $\dagger$ ). Methionine, on the other hand, revealed more organised fibre like morphology. The aromatic amino acids namely tyrosine, tryptophan and phenylalanine indeed showed fibril morphology as reported in previous studies (Fig. S1 ESI $\dagger$ ). ${ }^{6,7,8}$ The nature of interactions which lead to structure formation of Cys and Met were purported by co-incubating the assemblies in urea and mercaptoethanol which revealed the important role of hydrogen bonding and disulphide interactions in structure formation. The structure formation of Cys and Met were also assessed under varying $\mathrm{pH}$, concentration and time. The studies presented herein therefore might be of crucial interest to assess the etiology of many diseases caused by protein aggregation and moreover, provides a very simple and facile methodology for design of novel nanostructures through a bottom-up approach.

\section{Experimental Section}

All 21 L- amino acids were purchased from reference grade kit of L-amino acids offered by Sisco research laboratories (India) and used without further purification. The purity of all the amino acids procured was minimum 99\%. Deionized water was used for preparing all the solutions (Millipore). No additional organic solvent was added. Scanning electron microscopy (SEM) images were taken using a Nova Nano FEG-SEM 450 microscope (the accelerating voltage ranged from 5 to $15 \mathrm{kV}$ ). SEM samples were prepared on silicon wafers and 10 microlitre of $3 \mathrm{mM}$ solution of peptides were dispensed and dried at room temperature. The optical microscopy images were taken under Leica DM 2500 microscope with 40X magnification.

\section{Results and Discussions}

All $21 \mathrm{~L}$-amino acid solutions were analyzed within a concentration range of $1 \mathrm{mM}$ to $10 \mathrm{mM}$. All the solutions were prepared using only deionized water and no additional salts were added. The self-assembling properties of each amino acid were assessed by optical microscopy measurement. Of the 21 amino acids dissolved in four concentrations $1 \mathrm{mM}, 3 \mathrm{mM}, 6 \mathrm{mM}, 10 \mathrm{mM}$ 
only five amino acids namely, phenylalanine, tyrosine, tryptophan, cysteine and methionine showed fiber like assemblies. Figure 1a, 1b displays a SEM image of the structures formed by the $3 \mathrm{mM}$ solution of Cys and Met. The self-assemblies formed by both Cys and Met showed very long fiber like aggregates. The self-assembling behavior of Cys was particularly interesting since it showed neuronal fibril like morphologies with long fibers originating from single focus. It was found that these assemblies could be observed only in concentration range $1 \mathrm{mM}$ to $6 \mathrm{mM}$. Lowering the concentration below $1 \mathrm{mM}$ prevented self-assembly of any discernible structures. For the $3 \mathrm{mM}$ concentration, of the Cys, the dimensions of the aggregates were such that the diameter of the fiber varied from $200 \mathrm{~nm}$ to few micrometers and the length was on the order of several micrometers (see Fig. S2, ESI $\dagger$ ). These high aspect ratio structures were observed even when the concentration was increased up to $6 \mathrm{mM}$. However, above this concentration, crystalline precipitates of Cys were observed.

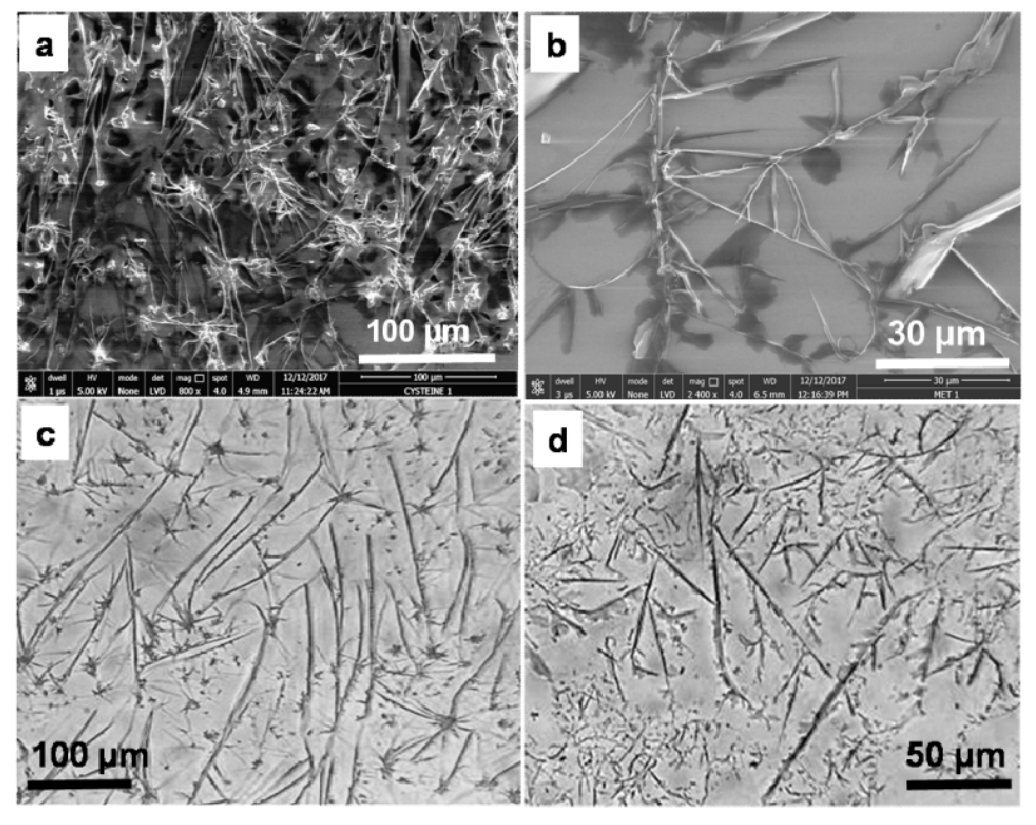

Figure 1:Self assembled structures formed by Cysteine and Methionine (a) SEM of 3mM solution of Cys, (b) SEM of $3 \mathrm{mM}$ solution of Met (c) Optical Microscopy image of $3 \mathrm{mM}$ solution of Cys (c) Optical Microscopy image of $3 \mathrm{mM}$ solution of Met.

Fig. 1b displays an analogous SEM image for solution prepared with methionine. It can be assessed that Met also assemble to fiber like structures, however, the fibers here appear more distinct and less aggregated as compared to Cys. The fibers albeit not straight but appear to be more organized and aligned. The length ranged in several micrometers with varying diameter from $200 \mathrm{~nm}$ to few microns. The fibers also appear branched. Figure 1c and Figure 1d are representative optical microscopy images of Cys and Met which also reveals a structure formation complementary to that observed in SEM and suggest the assemblies are indeed very big to be observed clearly by optical microscopy measurements. The Cys and Met structures werealso studied under varying $\mathrm{pH}$ (2-10). The structures were stable only under neutral conditions and disruption of morphology was assessed in slightly acidic and basic condition. (data not shown) 
Self-assembly is a spontaneous process of organization of molecules to well-ordered structures mainly driven by electrostatic interaction, hydrophobic interaction, hydrogen bonding, and $\pi$ $\pi$ stacking interactions. ${ }^{11}$ The formation of self-assembled structures by aromatic amino acids is mainly attributed to aromatic ring stacking $(\pi-\pi)$ of amino acids as envisioned earlier with aromatic functionalities. Thus Tyr, Trp and Phe assemblies may be attributed to pi-pi stacking and other possible interactions like van der Walls and hydrogen bonding like $\mathrm{C}-\mathrm{H}-\pi$ as discussed earlier. ${ }^{12}$ A non polar amino acid, like methionine which lack aromatic side chain may assemble to fiber like aggregates by hydrophobic interactions and possibly via backbone hydrogen bonding between nitrogen of amine and hydrogen of carboxylic group present in adjoining molecule of amino acid. ${ }^{11}$ Polar amino acids like cysteine may assemble mainly due to hydrogen bonding and thiol interactions. In addition, these molecules will also have tendency to form hydrogen bond with polar solvents like water. The role of water molecule in formation of such self-assembly as nanotubes/nanofiber is already reported. ${ }^{13}$

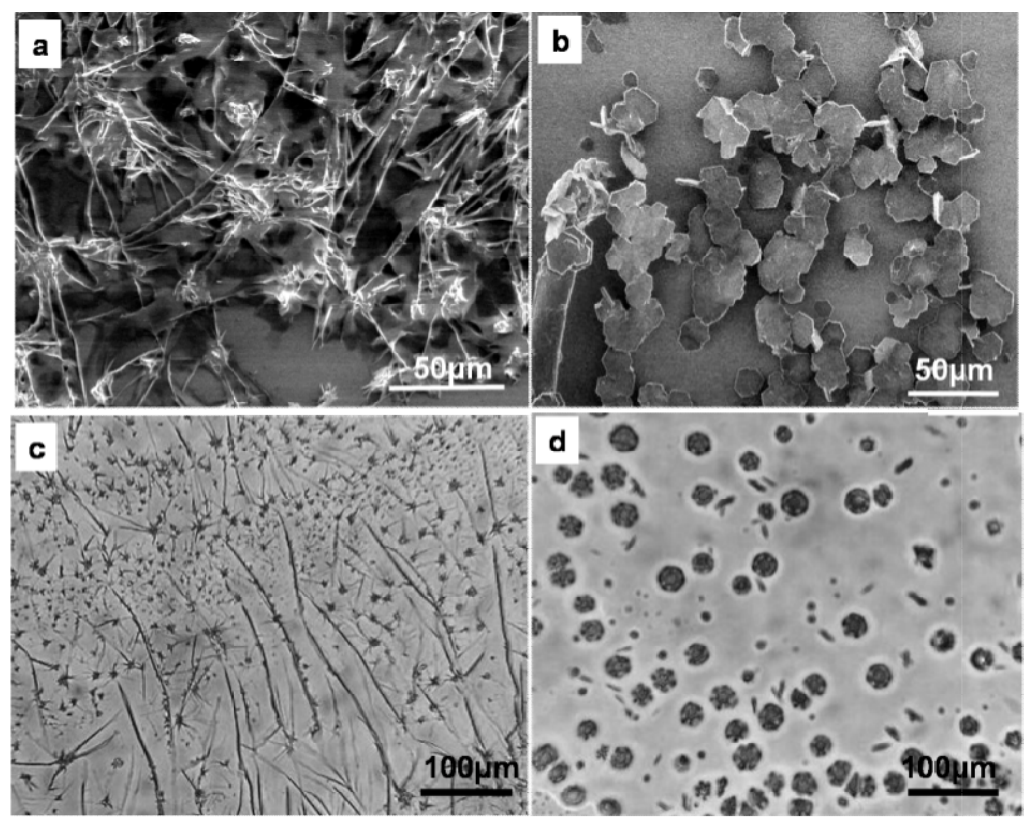

Figure-2.Microscopy images of cysteine withand without urea (a) SEM image of Cysteine alone (b) SEM image of cysteine with urea, (c) OM image of cysteine alone (d) OM image of cysteine with urea.

In our previous studies, we have reported a structural transition in self-assemblies owing to disruption of hydrogen bonding on co-incubation with urea. ${ }^{14}$ Thus, to probe the role of hydrogen bonding in structure formation of Cys and Met assemblies were co-incubated with urea. Figure 2 illustrates representative SEM and OM image of Cys after co-incubation with urea. A noticeable change in structure of Cys assemblies can be visualized. Methionine fibres were also disrupted completely on co-incubation with urea henceforth suggesting crucial role of hydrogen bonding in Cys and Met aggregation (Fig.S3, ESI†). Incubation with mercapto-ethanol lead to disruption of cysteine assemblies while methionine fibers remained intact suggesting a crucial role of - $\mathrm{SH}$ interactions in structure formation of Cys (Fig. 3). Further cysteine assemblies were also studied at $\mathrm{pH} 8$, the $\mathrm{pH}$ where $-\mathrm{SH}$ exist as $\mathrm{S}^{-}$and the disruption of assemblies could be assessed. 
Moreover, self-assembling propensity of cystine (dimer of cysteine where-SH exist as S-S) was also examined and it revealed lack of any structure formation further suggesting a crucial role of thiol interactions in Cysteine self-assembly (Fig 3).

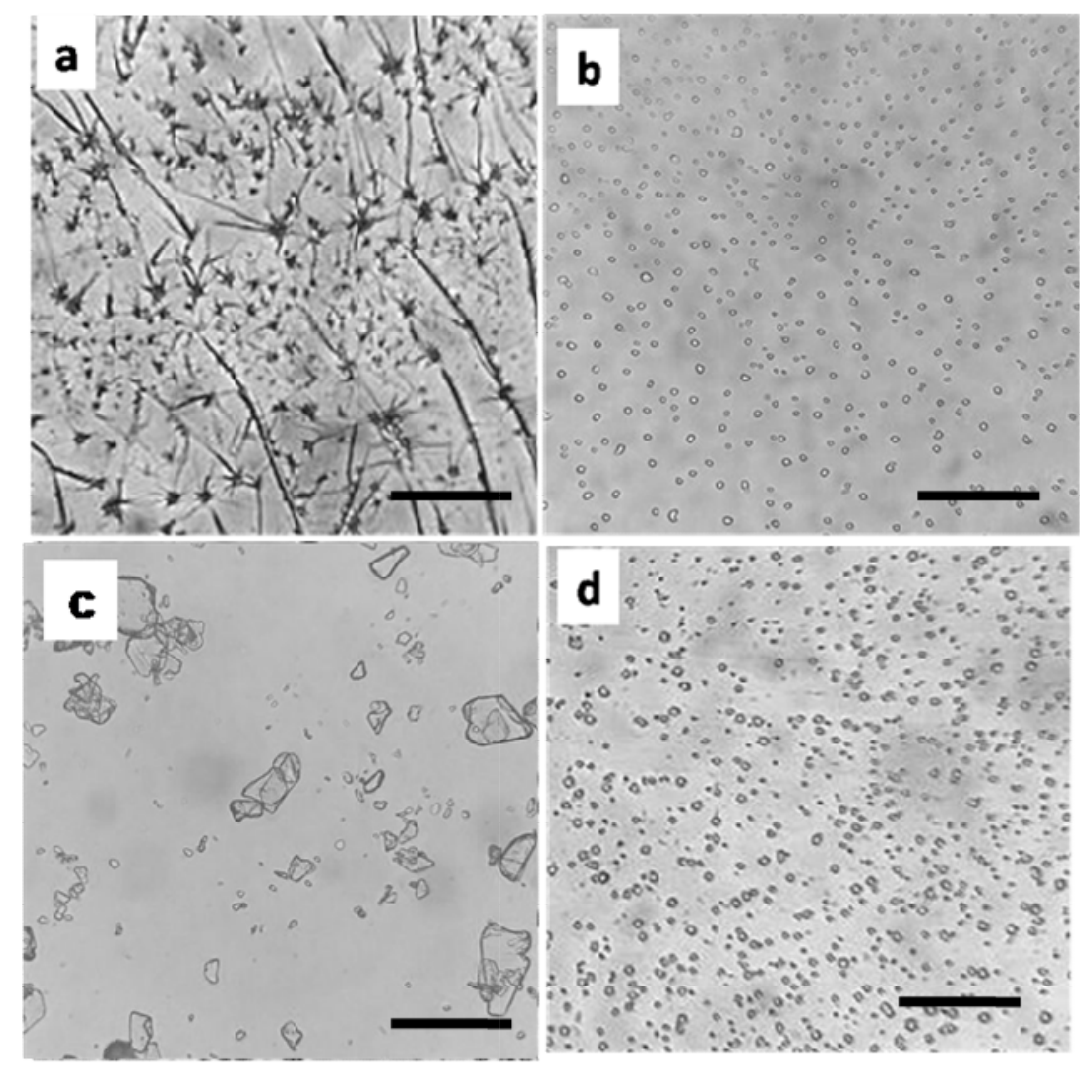

Figure-4: Optical Microscopy images in DI waterat 40X resolution (a) Cysteine (b) Cysteine at pH 8 (c) Cystine a oxidized dimer of cysteine (b) Cysteine + Mercaptoethanol. Scale bar $50 \mu \mathrm{m}$.

A recent literature report elucidate that cysteine possesses anti-amyloidogenic action ${ }^{15}$ while methionine triggers amyloid. Thus we were curious, to assess the effect of Cys and Met structure on fibrillation of amyloidogenic peptides. We coincubated short amyloidogenic dipeptide diphenylalanine (FF) which is used as a reductionist model of $A \beta(1-42){ }^{16}$ with Cys and Met fibres. While FF fibrillar assembly was completely disrupted by cysteine, addition on methionine revealed even more denser fibrillar structures (Figure 4, Fig. S4 ESI $\dagger$ ). Possibly, -SH group present in fibrillar structures of cysteine may interfere with aromatic stacking interactions. A recent report suggests that thiols can engage favorably with aromatic rings via $\mathrm{S}-\mathrm{H} / \pi$ interactions, within abiological systems and within proteins. The nature of this interaction was further examined by DFT calculations, IR spectroscopy, solid-state NMR spectroscopy, and analysis of the Cambridge Structural Database. These studies suggested that $\mathrm{S}-\mathrm{H} / \pi$ interaction are driven significantly by favorable molecular orbital interactions, between an aromatic $\pi$ donor orbital and the S-H $\sigma^{*}$ acceptor orbital (a $\pi \rightarrow \sigma^{*}$ interaction). ${ }^{17}$ Thus our studies, on interaction of cysteine fibres with FF (aromatic dipeptide), gives further insights into anti-amyloidogenic action of cysteine andimplicate that pi-pi stacking interaction between the rings of phenylalanine 
are interfered by thiol present in cysteine which may lead to disruption of fibres. It is also reported that methionine triggers amyloid proliferation which complements with the results obtained. $^{18}$

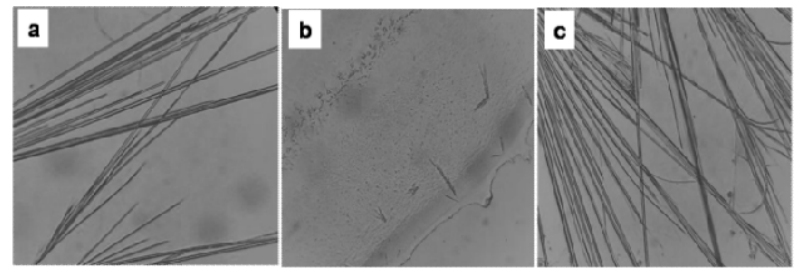

Figure-4.(a) FF alone (b) FF+ Cysteine (c) FF+ Methionine

\section{Conclusions}

In summary, a comprehensive study of self-assembling behavior of 21 amino acids was done. Five amino acids (Phe, Tyr, Trp, Cys and Met) revealed spontaneous structure formation in neutral aqueous solution. These results are highly significant since to the best of our knowledge till date, it is reported that only amino acids with aromatic side chains Phe, Tyr and Trp are capable of forming spontaneous assemblies. However, the studies reported herein, manifest for the very first time spontaneous structure formation by cysteine and methionine (amino acid lacking aromatic side chain).

\section{Acknowledgements}

We thank the SICART SEM facility and Dr. Sanjay Patel for assistance with SEM analysis.NG, BK, CP greatly acknowledge support from DST and SERB research grant (EMR/2016/003186) for funding and fellowships. DS thanks GSBTM for fellowship.

\section{Notes and references}

1 N. Habibi, N. Kamaly, A. Memic, H. Shafiee, Nano Today, 2016, 11, 41; T. Fan, X. Yu, B. Shen, L. Sun, Journal of Nanomaterials,2017, 2017; D. M. Leite, E. Barbu, G. J. Pilkington, and A. Lalatsa, Current Topics in Medicinal Chemistry, 2015, 15, 22, 2277; Kokkoli, E., Mardilovich, A., Wedekind, A., Rexeisen, E. L., Garg, A., \& Craig, J. A., Soft Matter, 2006, 2, 1015.

2 Yu, Zhiqiang, Quan Xu, Chenbo Dong, Su S. Lee, Liqian Gao, Yiwen Li, Mathew D'Ortenzio, and Jun Wu.,Current Pharmaceutical Design, 2015, 21, 4342; R. Huang, W. Qi, R. Su, J. Zhao, and Z. He, Soft Matter, 2011, 7, 6418; Sun, Leming, Zhen Fan, Yongzhong Wang, Yujian Huang, Michael Schmidt, and Mingjun Zhang., Soft Matter, 2015. 11, 3822; B. Akdim, R. Pachter, R. R. Naik.,Appl. Phys. Lett. 2015; 106, 183707.

3 M. Reches, E. Gazit, Science, 2003, 300, 625.

4 A Lakshmanan, S Zhang.C. A.E. Hauser,Trends in Biotechnology, 2012, 30, 155; J. J. Panda, V. S. ChauhanPolymer Chem.2014,5 , 4418.

5 E. Gazit, FEBS J., 2005, 272, 597.

6 Adler-Abramovich, Lihi, Lilach Vaks, Ohad Carny, Dorit Trudler, Andrea Magno, Amedeo Caflisch, Dan Frenkel, and Ehud Gazit, Nature Chemical biology, 2012, 8, 701. 
7 Perween, Shama, Balasaheb Chandanshive, Hema Chandra Kotamarthi, and Deepa Khushalani, Soft Matter, 2013, 9, 10141.

8 D. G. Babar, S. Sarkar, Applied Nanoscience, 2017, 7, 10.

9 Nelli, Srinivasa Rao, Rajan Deepan Chakravarthy, Yue-Ming Xing, Jen-Po Weng, and Hsin-Chieh Lin, Soft matter, 2017, 13, 8402; P. Koley, Pradyot, and Animesh Pramanik, Advanced Functional Materials, 2011, 21, 4126; Koley, Pradyot, and Animesh Pramanik, Journal of Materials Science, 2014, 49, 2000.

10 N. Gour, S. Verma, Soft Matter, 2009, 5, 1789; Gour, Nidhi, Jancy Nixon Abraham, Mohamed Chami, Axel Castillo, Sandeep Verma, and Corinne Vebert-Nardin, Chemical Communication, 2014, 50, 6863;Kedracki, Dawid, Sergey K. Filippov, Nidhi Gour, Helmut Schlaad, and Corinne Nardin, Macromolecular Rapid Communication, 2015, 36, 768.

11 S. Toksoz, H. Acar, and M. O. Guler, Soft Matter, 2010, 6, 23, 5839; Ghadiri MR, Granja JR, Milligan RA, McRee DE, Khazanovich N., Nature, 1993, 366, 324.

12 Mishra A, Chauhan VS.,Nanoscale, 2011, 3, 945; Reches M, Gazit E.,Curr Nanosci., 2006, 2, 105; Meital R, Ehud G., Physical Biology., 2006, 3, S10.

13 Fujibayashi K, Hariadi R, Park SH, Winfree E, Murata S, Nano Letters, 2008, 8, 1791; Begum A, Tripathi KM., Sarkar S., Chem Eur. J., 2014, 20, 1.

14 Nidhi Gour, Dawid Kedracki, Ilyès Safir, Kien Xuan Ngo and Corinne Vebert-Nardin,Chemical Communications, $2012,48,5440$.

15 Zaman, Masihuz, Syed Mohammad Zakariya, Saima Nusrat, Tajalli Ilm Chandel, Syed Musthapa Meeran, Mohammad Rehan Ajmal, Parvez Alam, and Rizwan Hasan Khan, Int. J. Biol. Macromol., 2017,105, 556.

16 S. Brahmachari, Z. A. Arnon, A. Frydman-Marom, E. Gazit, and L. Adler-Abramovich ACS Nano, 2017, $11,5960$.

17 Christina R. Forbes, Sudipta K. Sinha, Himal K. Ganguly, Shi Bai, Glenn P. A. Yap, Sandeep Patel, and Neal J. Zondlo, J. Am. Chem. Soc., 2017, 139, 1842.

18 Tapia-Rojas, Cheril, Carolina B. Lindsay, Carla Montecinos-Oliva, Macarena S. Arrazola, Rocio M. Retamales, Daniel Bunout, Sandra Hirsch, and Nibaldo C. Inestrosa, Molecular Neurodegeneration, 2015, 10, 62 